\title{
Usability perception of different video game devices in elderly users
}

\author{
Ramón R. Palacio ${ }^{1} \cdot$ Christian O. Acosta $^{1} \cdot$ Joaquín Cortez $^{2} \cdot$ Alberto L. Morán $^{3}$
}

Published online: 22 October 2015

(C) The Author(s) 2015. This article is published with open access at Springerlink.com

\begin{abstract}
The purpose of this study was to evaluate the usability perception of seniors on video game use with different control devices. Twenty-four seniors participated in the study (12 women, 12 men, 69 years old in average) and eight children ( 8 years old in average). The setting included the use of two Kinect motion sensors, three computers, three projectors, three video cameras, two audio devices, the Angry Birds and Happy Sky videogames, one Xbox 360 console, a Nintendo Wii device and a Touchscreen configured so that participants could play the games in senior-child pairs. Data were collected according to user friendliness, experience and social interaction. This study suggests that video game control devices for seniors should be adapted to compensate for their function, sensor and cognitive limitations.
\end{abstract}

Keywords Elderly · Usability · Video game devices

Ramón R. Palacio

ramon.palacio@itson.edu.mx

Christian O. Acosta

christian.acosta@itson.edu.mx

Joaquín Cortez

joaquin.cortez@itson.edu.mx

Alberto L. Morán

alberto.moran@uabc.edu.mx

1 Unidad Navojoa, Instituto Tecnológico de Sonora, Ramón Corona S/N, Col. ITSON, 85860 Navojoa, Sonora, Mexico

2 Unidad Nainari, Instituto Tecnológico de Sonora, Antonio Caso 2266, 85130 Ciudad Obregón, Sonora, Mexico

3 Facultad de Ciencias Ensenada, Universidad Autónoma de Baja California, Km 103 Carretera Tij-Ens, Ensenada, Baja California, Mexico

\section{Introduction}

Playing videogames requires concentration, memory, coordination and reaction speed. As video games reinforce these abilities and skills, they seem to be beneficial for senior users. Video games can improve attention, eye-hand coordination, fine motor skills, short-term memory, problem-solving and reaction-time speed to new situations. Also video games, when involving different people in the game, can contribute to emotional well-being. As video games produce positive affection, they are associated with mental health improvement. However, only a few studies have been published on the effects video games have on seniors. These studies suggest the possibility of behavioral, cognitive and emotional effects [1]. Physically-based video games can motivate senior users to exercise and, as an additional benefit, their social nature can help on solving the isolation problem in some of them.

Even though there are few studies on the social and physical effects of physically-based video games in the senior population, the use of Nintendo Wii has become popular in physical rehabilitation, depression symptoms reduction and cognitive development in this population with a positive impact on their psychological and physical well-being, promoting a higher enjoyment of the social aspects of video games. However, there is inconclusive evidence on the physical and health benefits from the use of video games based on physical exercise for seniors, as well as their effects on the psychological aspects [2].

Participating in activities helps seniors to feel better and stay healthy as they recognize their capability to move and create something new. According to different studies, moderate and regular exercise can be helpful to fight depression in senior citizens. Also, technology helps seniors to keep in touch with family and friends, provides 
home safety, and assists and facilitates their health care. Also, technology offers new incitement in their life and facilitates access to information. Different studies confirm the role of technology in increasing social interaction, self-esteem, life satisfaction and personal autonomy. Based on physical activity, social interaction and cognitive aging's positive relations, it is important to research on how to use digital technology to foster seniors' wellbeing through physical activity, entertainment and social interaction [3].

As a result of the enormous progress on learning technology, digital games have become feasible and viable learning tools. However, due to the impact of aging on human-computer interaction aspects, the development of more user-friendly interfaces is necessary to solve the illiteracy problem and psychomotor obstacles in many senior users. As people age, there is a reduction not only in their general ability to maintain attention but also in their sensory motor skills. The main limitations of the elderly in terms of health that must be considered when using a computer are [4-6]: (1) Sight: reduction in the field of vision, ability to distinguish small details, process visual information and adjust to darkness; (2) Hearing: reduction in the ability to hear certain sound timbres or distinguish certain frequencies; (3) Mobility: slower response times, reduction in fine motor skills and greater fatigue; (4) Cognition: decrease in attention span, specially if there are distractions, short-term and working memory loss.

Besides, older people take longer to perform certain activities and to read instructions and textual information, they make more mistakes, they forget the point of the activity they are performing, they are more often confused by or do not understand technical language, they are reluctant or refuse to do something they think will cause system failure, and they get more upset and often blame themselves if something goes wrong.

Fisk et al. [7] conducted different focus groups with senior citizens and found that more than $50 \%$ of the problems reported by participants while using technology were related to user friendliness and that this can be solved with design improvements or training. Input and output devices are a sensible aspect in particular, because they imply interaction with the sensor or perception system of the user.

As stated earlier, senior users suffer from age-related changes that make difficult for them to use a product or service; that is, they face usability problems. According to [8], a product or service is usable if "the user can do what he/she wants to do the way he/she expects to be able to do it, without hindrance, hesitation or questions." Also, according to these authors usable products or services have specific features or attributes, including usefulness, efficiency, effectiveness and learnability, among others.
Furthermore, these authors identify some characteristics related to usability that are particularly important when considering senior users:

1. Learnability the difficulty is to learn to use a device and to understand and integrate instructions. The time required to correctly complete a task and the results obtained in a certain amount of time are possible measurements of learnability.

2. Efficiency technological applications satisfy the user's needs to a certain degree so that they avoid time loss, frustration and dissatisfaction. Efficiency can be measured by the execution of an experimented user in a specific task.

3. Errors how easy senior users can produce errors with a product and how easy these errors can be solved by the users.

4. Satisfaction user's attitude and adoption of technological applications can be influenced by the pleasure associated with its use.

Additionally, age-related neuromuscular changes entail that muscles in seniors do not respond in the same way as in young adults. These muscular aspects combined with cognitive and perceptual challenges affect the execution of fine motricity tasks. These motricity tasks include aiming, grapping and reaching. For instance, it is not easy for senior users using the computer mouse and, at the same time, making a double click and selecting objects on the screen. Therefore, it is very important to ensure the usability of an interactive system. Because of this, the design of a technological device for senior users must be based on familiar aspects of the activity, and also, it has to reduce learning efforts, minimize interface elements and maximize interface consistency. However, senior citizens are little familiarized or used to computer technology and possibly they do not have the required basic knowledge to interact effectively with it. For these reasons, the information required to correctly execute a task must be immediately visible in the interface for the senior users to interact with, rather than having to base their actions on intuition or memorization of long sequences of operations [3]. Thus, the following is the research question: What is the perception of Usability of seniors using different types of control devices in video games?

Comparing seniors experience related to the use of control devices of Xbox, Wii, Touchscreen and Kinect could help us answering such research question. In this context, the main objective of this study is to evaluate the perception of usability of senior citizens while playing video games using different control devices to identify a set of ideas to support the development of technology appropriated to this population. 


\section{Related work}

This section presents several research works that have addressed the issue of elders' perceived usability to the video games, which are presented as follows.

A study conducted by [9] has the purposes of (1) understanding the elders' perceived usability and dependability of one embodied interactive video game system; (2) understanding the flow experiences of the elders while playing on the system; (3) exploring the relations between the elders' perceived usability, dependability of the system and the flow experiences. There were 30 elderly (above 60 years old) participants, 14 male and 16 female. They were from three different settings: rural community, elementary school volunteer groups and a nursing home for elderly. The embodied interactive video game system supported the physical interaction where the player's body should become the interface to the system. The players could move their bodies, wave their hands or shake their heads to manipulate the digital elements in the game system. In other words, by being based on capturing body movements with an embedded webcam, this game system relied on the physical interactions of the players' movements. The first game was about categorizing the food for health. The second game was about identifying the symbols of Asian or European countries. The third game was a kind of race competition. Related to the first purpose of this study, the major method to collect data was by interview. Observation was used for triangulation. The interviews addressed: (1) the difficulty to learn to play the game; (2) the time needed to perform a previously experiences task; (3) the tendency to induce errors; (4) safety, confidentiality; (5) the extent to satisfy the users; (6) the pleasure derived from usage; (7) the integrity and maintainability. Related to the second purpose of this study, the measurement of flow experiences was done by observing the expressions and gestures of the subjects while playing. Interviews were used to obtain the feelings of the subjects while playing. Feelings included clear goals, degree of concentration, loss of the feeling of self-consciousness, immediate feedback, balance between ability level and challenge, sense of personal control, intrinsically rewarding and action awareness merging. Related to usability experiences, the elders answered "It is very easy to learn to play the game." The researcher could feel their confidence and pleasure in playing from their expressions and gestures. Related to flow experiences, the elder said "I enjoy the challenge, I lose the feeling of self-consciousness during the process of recalling." Finally, the usability and dependability of the system helped the elders with less computer experience and prior knowledge to experience the flow.
In addition, one of the purposes of a study conducted by [10] was to investigate the extent of affective responses and satisfaction of elderly users when using the Nintendo Wii. Thirty-one participants aged 50 years and above took part in the study. More than $50 \%$ of the participants were above 60 years old. Two-thirds of the participants were females. Constructs of perceived usefulness, perceived ease of use, behavioral intention, perceived health value, perceived affective responses, perceived sociability value and perceived satisfaction were measured. Cronbach's alpha for all the constructs were above 0.7. Results indicate that perception about the "perceived ease of use" has a significant relationship with "perceived usefulness." The relationship between perceptions of "perceived health value" and "perceived usefulness" was found to be significant. Also they examined the relationship between the "perceived ease of use" and "perceived health value" and found a significant positive relationship between the two constructs. Furthermore, "perceived usefulness" in elderly perception has a weak significant relationship with "perceived satisfaction." However, the study shows a strong significant relationship between "perceived usefulness" and "perceived affective responses," and a moderate significant relationship between "perceived affective responses" and "perceived satisfaction." A strong support is also shown specifying a significant relationship between "perceived usefulness" and "perceived social value." However, there is no significant relationship between "perceived social value" and "perceived satisfaction." "Perceived affective responses" mediate the influence of "perceived usefulness" on "perceived satisfaction," and "perceived satisfaction" is a significant predictor of "behavioral intention" for adopting the Nintendo Wii. Authors conclude that perceived ease of use and perceived health value were determinants of perceived usefulness. Perceived affective response mediates the influence of perceived usefulness on satisfaction, leading to elderly users' behavioral intention to use. However, there is no significant relationship between perceived social value and perceived satisfaction, implying that perhaps personal enjoyment with the Wii is more important than having the opportunity of playing with others. Apart from the entertainment value, the study shows that the Wii games invoked positive affective responses. The involvement with the Wii games could provide alternative ways to keep the elderly active.

In the same way, [8] presents a project to promote physical and social activity among members of group homes for the elderly using Wii Bowling League. Thirty participants were observed (between 60 and 94 years old) for 10 sessions. All sessions were videotaped in order to identify changes over time on their performance with the game. For video analysis researchers took into account the body posture, gestures, layout of the room, the sound 
qualities of speech, game elements and language. As a result they found that the physical movements that are a necessary part of playing the Wii help to define a "sacred" space around the game with its own sense of decorum. This space was considered for senior citizens as a place where they can learn new technical literacies, make new social connections with peers and take ownership of the communal spaces in which they live. Thus, this work concludes with key lessons for encouraging appropriation and empowerment for older people through game play in communal housing settings.

Furthermore, in [11] authors show that game design for older people could help them to be more socially active in their communities. With this, the authors pretend to understand the necessity to design a simple, socially enjoyable and entertaining new game for older adults. Thus, the authors present multiple design cases that are considered as an initial research in game design focused in older adults.

Concerning the first case, the study informs on the evaluation and design of an Augmented Tabletop Game that was especially created for seniors in order to provide leisure and fun; it is played among four players. The aim of game is for players to find their way across a city map. Players are instructed to visit three famous monuments in a beforehand specified order. The game was evaluated with eight voluntary participants, 5 males and 3 females, aged 65-73 years. The two separate sessions were videotaped. The evaluation was conducted in a laboratory since the augmented tabletop system could not be easily moved. The participants played the game and then responded to a Game Experience Questionnaire (GEQ). The authors present two important findings: (1) the game playing experience of the elderly can be enhanced by digital tabletop games, as technology is latent in them and hence dynamic game behavior can be incorporated, leading to easy interaction, and (2) adaptive multimedia plays an important role when designing an engaging and enjoyable tabletop game for the elderly.

Regarding the second case, it describes a Walk 2 Win Game that had as objective to motivate older adults to socially participate among themselves. The game has two levels (easy and hard) and two modes (single or multiplayer) and was installed in four smartphone. The game was evaluated to determine whether the older adults understood the game rules and whether they perceived the game as a fun. The game was evaluated in individual and in multiplayer mode with eight participants $(5$ men and 3 women, age range from 67 to 78 years, mean value 71 years). As the researchers concluded that the game should include as many players as possible, the game rules should be minimal and the game should be simple and fun.
Finally, the third case describes the design of a social active mobile game for grandparents and their grandchildren called Grand Game. The aim of this game was to teach grandparents and their grandchildren to develop healthy eating patterns. The purpose was to explore what the differences and similarities between elderly and children are during game play. Eight older adults evaluated the game; they responded a brief questionnaire after they had the experience with Grand Game. In this case, the importance to have and stimulate a healthy eating pattern for these target groups is highlighted.

Another work [12] presents a platform based on game consoles (Nintendo Wii) that demand body movements to control the player's performance in a highly motivating context. This platform was called SUW unit. The SUW has only four clearly labeled buttons, each one of them representing one possible sport game. When the user presses one, the unit automatically performs all the necessary configurations to start a game. By doing so, the person simply needs to exercise itself while playing, without needing to repeatedly navigate through the console menus by means of the not-elderly-conceived remote control. The objective of this study was to simplify the use of new technology for senior users, particularly regarding mechanical and functional aspects.

For analyzing the acceptance and functionality of the SUW unit, two test sessions with different groups of seniors were performed. For the first one, 48 volunteers (16 women, 32 men, age range from 59 to 85 years, mean value 68.4 years) were recruited. The second test session was performed with 10 residents of a nursing home (6 women, 4 men, age range from 72 to 96 years, mean value 82.9 years). For each session, the task consisted of starting a game, play for a while and shutting the system down. The evaluation of the experiment was carried out by observing how the volunteers performed the assigned tasks and also by responding a specially designed questionnaire. As results of this work, the researchers conclude that the SUW unit, by simplifying the usability of such systems, would boost acceptance. The tests indicated the need to focus the system's improvement on elderly people that would most profit of its benefits according to the demographical change trend.

Other applications identified also address cognitive issues. For instance, on the one hand [13] highlights an increasing need for more appropriate methods in the design of technology for seniors. This research addresses digital television and proposes the implementation of an induction memory system and a maintenance program for people with Alzheimer as other minor cognitive deterioration. To do so, it promotes interactive television sets that allow for favorite channels to be programmed and show a description of them. 
On the other hand, in [14], a video game design was presented. The video game so-called Song of North mixes physical and virtual reality. The game is played in a cemetery (interface), the player becomes a sorcerer or shaman who can recognize different analyzed sounds during the game and uses a drum to produce them. The design solution emphasizes that the player can move throughout the physical surroundings simulating the movement in the game. In this study, a set of design requirements was formulated, where the main findings were on what requirements were needed and the need of having creative designers.

An additional topic addresses the use of technology to support senior citizens' emotional problems. An example of this is [15], which results from the project "Age and Experience." This study explored ways in which technology can strengthen bonds among different generations. It describes the results from a two-year study in a retirement house and develops a notion of inter-generational participation through ludic and playful systems that stimulate joy and curiosity. Also, this study describes innovative ways to involve older generations through digital "curiosities or cabinets of wonders" as Bloom, Tenori On and Google Earth. One of the main problems identified is that people living in retirement houses tend to fall into depression and to be lonely because they are away and separate from family and friends. Usually, requirement analysis shows a senior citizen as a being or entity full of disabilities rather than as an individual. The experience during this study shows that seniors are not static entities and that their abilities vary day after day.

Additionally, Barrington et al. [16] present an operational prototype that represents aspects of the affective functioning in a musical effect code. During this study, a specific case was studied, aiming: (1) to investigate the emotional effect that music produces in a human being; (2) to create a functioning prototype that provides a musical atmosphere in accordance with what it is reflected by gestures of the user's face.

Finally, we found the topic of the physical problems seniors may acquire through the years. Let us consider the project "Through the looking glass," which consists of creating a graphical representation of a place visited and, then, making a graffiti according to their experience or life lesson. It uses GPS technology to locate places and objects that can be adorned with "graffiti." That is, this technology can be used to locate this type of stories, and visiting these places with our imagination, etc.

As was noticed, different efforts have been made to understand how video games can help senior citizens through time. However, no studies that provide direct results of senior interaction with control devices were found. That is, there was no evidence whether the devices are customizables for elderly people to accomplish an adequate performance during video game interaction, and whether this indicates that the senior citizen is willing to interact with this type of technology. Considering the aforementioned evidence, the importance of this study relies on collecting data that help understand how senior users can better interact with video games using different control devices.

\section{Method}

The research design used for this study was nonexperimental and explanatory [17].

\subsection{Participants}

In total, 33 users participated in the study. Of them, 24 were senior users with ages ranging from 60 to 79 years $(M=69, \mathrm{SD}=5.78), 12$ female and 12 male. The rest of the participants were 8 -year-old children.

\subsection{Materials}

To conduct the videogame test, two Kinect motions sensors, three computers, three projectors, three video cameras, two audio devices, the Happy Sky videogame software, the Angry Birds videogame (classic version), one Xbox 360 console, a Nintendo Wii device and a Touchscreen were employed.

Also, a measurement device was used to interview the participants (individually) and to measure their perception. A sample of the type of questions asked per usability characteristics is:

\section{Learnability}

1. Do you think this game was difficult to learn to play?

2. If you had this game at home, would you play it?

\section{Satisfaction}

3. Did you have a good time playing?

4. Do you think time passed by too quickly?

5. What would you consider that the video game must have so that children may get involved and enjoy playing?

6. Did you like to play in turns?

7. Did you like to share your experience with your peers?

8. Would you like to compete? 
Also, a questionnaire was used for the group discussion with learnability and satisfaction items. The set of questions is presented as follows:

1. Was it difficult to interact with the video game?

2. Did you feel uncomfortable and uneasy while playing?
a. Why?

3. Could you master this type of games with some practice?

4. Would you like to play for competition or just for fun and entertainment?

5. Did you have a good time playing?

\section{a. Why?}

6. Do you believe video games are a good entertainment activity for a senior citizen?

7. Would you like to play different video games?

a. If the answer is Yes:

i. Which type?

b. If the answer is No

i. Why you would not like it?

8. Which method or modality made you feel that time ran faster?

\subsection{Procedure}

Two studies, described below, were conducted:

\subsubsection{Study 1}

In a group of nine senior-child pairs, couple interaction was evaluated with the Happy Sky video game [18]. To evaluate it, seven steps were followed:

1. An adequate space was set, a computer with the Happy Sky software and the Kinect motion sensor was installed.

2. The participants were informed of the intention and objective of the study (knowing whether senior users could adopt such technology). Their participation and the way it would be developed were explained.

3. A brief explanation of the Happy Sky was introduced. The objective of the game is to finish two levels: The first level (i.e., flying a kite) has the objective of familiarizing the user with the sensor allowing him/her to move his/her hands freely. The second level (i.e., catching a dragon) requires collaborative work, and thus the two players must use two nets to simultaneously capture a dragon. The dragon moves freely at different speeds. To capture the dragon, it must be taken into a cage, which appears when accomplishing the collaborative task.

4. Children were asked to select a senior citizen as a partner.

5. A 5-min training (in average) was done, per couple.

6. Every couple was asked to accomplish the videogame's objectives (as described in step 3). Every couple's behavior was videotaped during the time approximately (20 $\mathrm{min})$.

7. Senior users were interviewed, and audiotaped, to document their experience.

8. The interviews were conducted in groups (60 min) and individually (10 min). Participants expressed their ideas as open as possible.

9. Data were extracted from the interviews using open, axial and selective data coding processes, which are used in grounded theory [19]. This process initiated with open coding in which data were divided into categories (recurrent concepts) and subcategories. Subsequently, it continued with axial coding during which the relationships between categories and the possible causes of their behavior were examined. Finally, for selective coding, the categories were integrated and conclusions were obtained.

\subsubsection{Study 2}

In a second group, the interaction of 15 seniors with the Angry Birds video game (classic version) was evaluated. Four different platforms (i.e., modalities) were used to play the game, including Xbox 360, Nintendo Wii, Touchscreen and Kinect. To conduct the evaluation, six steps were followed:

1. A brief explanation of the logic and control of the game.

2. The participants were divided into two groups, one including seven users and the other including eight users. Every group represented an interaction modality. The session consisted on Nintendo Wii for the 7-users team and Xbox 360 for the 8-users team. They followed these steps:

- First, there was a 15-min training session per team. Each team could play one game as training.

- Each member tested his/her ability and skills in level 1 of the game only.

- After finishing playing in the assigned modality, the teams switched modalities.

3. A 15-min break was granted so that participants could eat something and relax before continuing with the next part. 
4. After the break, participants played on the other modalities (the Touchscreen and Kinect) following the same methodology as in step 2.

5. A discussion group was created (for approximately $30 \mathrm{~min}$ ) with participants sharing information on their experience with the video game and their interaction with control devices. Information was gathered using the questionnaire described in the "Materials Section".

6. Finally, two of the videogame sessions filmed were analyzed. For that, there were two usability characteristics selected to measure the interaction with the video games, as follows:

\subsubsection{Efficiency}

Control time (hold-aim-fire/ throw):

Total time spent from the moment the user takes control captures the bird aims and throws.

\subsubsection{Errors}

Effective

Total number of effective throws during throws:

Missed throws: the game. Total number of missed throws during the game.

\section{Results}

\subsection{Study 1}

Regarding the results of the interaction of senior users and children with the Happy Sky videogame, they measured considering the following metrics: (1) Learnability, (2) Satisfaction (3) Errors and (4) Efficiency.

Concerning Learnability, participants demonstrated the ease and ability of using the video game, measured through the game's management and interaction's simplicity shown by participants, both older adults (OA) and children did not have any problems while manipulating their avatar/character (in this case the kite). Some of the answers were:

“...Well, it wasn't too difficult for me, it was easy..." [OA4].

“...Can you imagine, it didn't take me two minutes, there were seconds to catch the dragon..." [OA5].

“...Oh, it was too short, but productive because we captured the dragon quickly..." [OA5].

The ease of use, an element from learnability, was present during the game with Kinect because players could use the sensor without difficulties. Players could understand the way to use it and achieved the game objective.
Also, it was noticed that senior users could experience the ease of use of the video game because it was attractive to them.

“... Of course, because it distracts me, instead of being in bed with bad thoughts, I would use it twice or three times a week..." [OA4].

“...it is clear, it stimulates your brain, it is good to makes something not just to do something, it makes us think and solve problems, to see who solves it quicker and which way..." [OA7].

"...yes, just the way they do these video games, more positive, more productive and positive for us and for the children, not just a violent mentality..." [OA8].

Senior participants expressed how easy it was for them and they considered using videogames as a leisure activity. This can be confirmed because $100 \%$ of the couples achieved objective 1 "free fly," while $78 \%$ of them achieved objective 2 "Catch the dragon." It should be noticed that couples had the challenge to achieve both objectives.

As of Satisfaction it was shown that participants were satisfied on playing the videogame with Kinect because they were concentrated while playing, paying attention to the screen, to their movements and teammates; also they were careful not to step out of the Kinect sensor area. Some of the comments were:

“...children are an inspiration, they transmit us the game, because of their age they transport us to our youth..." [OA5].

"...Just the way time flies, it is very nice, it is entertaining for us..." [OA1].

"...I feel motivated, this is something I didn't know and that I could do without any problem..." [OA1].

In this classification, there were certain points where participants demonstrated that during the test some of them felt anxious and it was difficult for them to pass to the next level, or were desperate because they took longer to accomplish the objectives and it was a little tiring. On the contrary, other participants did not show anxiety because they finished the game without difficulty. Some comments regarding this matter were:

“...We wanted to catch the dragon but we couldn't

do it, we struggled..." [OA6].

“...I got tired my hand hurts..." [OA3].

Continuing with this classification of participants, it was observed that they had a great time by using the video game, in addition to sharing time with other people, which was very favorable for their mood. Some supporting comments are shown below: 
“...I remember my childhood a bit, the game was very satisfying, it is nice..." [OA1].

“...Yes of course, I felt very well..." [OA3].

"...I am excited because I did it in less than 2 minutes; it took us seconds to catch the dragon..." [OA5].

Enjoyment was an important element while playing as participants were happy while interacting because they were busy and concentrated during their turn. Some comments were:

“...I was learning, I was even laughing, I never thought I would be playing..." [OA8].

“...When you are having a great time, time flies always so quickly..." [OA3].

"...It was really fast, we finished it right away..." [OA2].

In this same classification, it was observed that attention was a factor present during this test because participants were focused on the game, on the screen and the sensor.

Participants were not interested in using video games as a way of competition but as a way of spending time with family or friends. Some comments were:

"...Winning or losing, it is the same for me..." [OA4].

“...I need to play it first to understand it, then I'll compete..." [OA2].

"...I think I will just get some entertainment, and then I will compete to see what results I can get..." [OA3].

Moreover, participants wanted to cooperate because it was indispensable to communicate with their teammates to accomplish the objective. Some comments were:

"...it is motivating to spend a good time..." [OA3].

"...spending a good time with my grandchildren,

before with my children, now with my grandchildren..." [OA5].

"...despite the age difference, everything was fine because there was more communication between both of us..." [OA7].

Communication was an important element while playing with Kinect to accomplish the objective of the game and interact with the teammate. This element is reflected in the amount of interactions made by the participants. When having the opportunity to play, participants enjoyed the interaction not only with the Kinect sensor and the game but also with other people, because playing with a couple was required. During this process, the mood and spirit of senior users were getting higher because of their interaction with children. It is necessary to mention that only two couples had a family relation (grandfather-grandson), and that they finished all levels faster than the rest. This could happen because the numbers of interactions were higher than for the other couples who did not know each other.

Results from Study 1 were important because this study helped seniors and children to interact, not only while playing but also as teammates during the test, as with the rest of the people around them. This could happen because they noticed they could manipulate this technology and interact with other people at the same time. Also, it is a way to keep them occupied performing certain movements and developing an answer and reaction.

\subsubsection{Study 2}

Regarding Efficiency and Errors as usability characteristics, results were obtained from the Angry Birds video game. Descriptive statistics obtained from Xbox show it as the device with an average of $2.4(\mathrm{SD}=0.964)$ as of $E f$ fective Throws; according to the average points, this device was more effective. It has to be emphasized that this was the device with the least number of Missed Throws (average $=1.25, \quad \mathrm{SD}=1.342$ ). Given the aforementioned results, Xbox looks like the favorite device to interact. As Kinect and the Touchscreen get a similar level as of Missed Throws, both obtained an average of 1.36 (SD $=1.151$ and $\mathrm{SD}=1.008$, respectively). The Wii obtained an average of 1.86 (SD = 1.099), which made it the least effective device to play the videogame. Regarding Effective Throws, Kinect is the second device with which participants achieved an average of $0.79(\mathrm{SD}=0.426)$ and Touchscreen was a device with which senior users had higher difficulty to achieve effective throws, obtaining an average of 0.57 ( $\mathrm{SD}=0.514)$. On the other hand, Wii had an average of 0.64 ( $\mathrm{SD}=0.497$ ) on effective throws.

As of effective and missed throws, Xbox averaged the highest effective throws and Wii the highest missed throws.

As of control time, Xbox gave participants the opportunity to take more time on calculating movements and take control over the game, so that the participant invested part of that time on how his/her movement would be and applying it. As the Xbox allowed more time (average $=27476.56 \mathrm{~ms}$ ), a participant could decide the moment to make a play (throw). This indicates that time invested allows a higher probability to hit and obtain an effective throw: Participants achieved a better performance with this device.

Therefore, it is assumed that control time has a direct influence on the throw, that is, a longer control time means more effective throws. Kinect and Wii have similarities when compared; both of them did not require much control time, which is why it was inferred that there were higher 
Table 1 Multiple comparisons

\begin{tabular}{lllllll}
\hline Measurement categories & \multicolumn{2}{l}{ Average difference among devices } & & & \\
\cline { 2 - 7 } & Xbox kinect & Xbox touchscreen & Xbox Wii & Kinect touchscreen & Kinect Wii & Touchscreen Wii \\
\hline ET-effective throws & $1.652^{*}$ & $1.866^{*}$ & 1.795 & 0.214 & 0.143 & -0.071 \\
MT-missed throws & -0.107 & -0.107 & -0.607 & 0.00 & -0.5 & -0.5 \\
CT-control time (ms) & $13728.063^{*}$ & $22461.705^{*}$ & $16170.063^{*}$ & 8733.64 & 2442 & -6291.64 \\
\hline
\end{tabular}

$* p \leq 0.05$

probabilities on missed throws. However, it is important to emphasize that the Wii control had more missed throws (average $=1.86$ ), with an average control time of $11306.50 \mathrm{~ms}$, while Kinect obtained an average of $13748.5 \mathrm{~ms}$.

The Touchscreen registered less time for the control time, $5014.86 \mathrm{~ms}$ and with less effective throws getting an average of 0.57 .

In Table 1, the average differences among every device are shown according to every category. It can be seen that significant differences exist. A single factor variance analysis was performed with a $p \leq 0.05$ to establish significant differences in different comparisons.

As of effective throws, $\mathrm{F}(3)=28.78, p<0.001$, it is shown that when comparing Xbox versus Wii, the Touchscreen and Kinect, there are significant differences. However, when comparing the Kinect to the Touchscreen and Wii, there are no significant differences, as when comparing the Touchscreen and Wii.

For missed throws, $\mathrm{F}(3)=0.79, p>0.05$, when comparing the Xbox versus Kinect, the Touchscreen and Wii, no significant differences were identified, as in the case of the Touchscreen and Wii.

Finally, regarding control time, $\mathrm{F}(3)=8.47, p<0.001$, when comparing the Xbox versus Kinect, the Touchscreen and Wii significant differences were identified. However, when comparing the Kinect with the Touchscreen and Wii, no differences were identified, compared to the Touchscreen and Wii.

\section{Insights}

The results of the two studies previously presented provide insights that can offer guidance for developers to design new forms of videogames interactions for senior users.

Regarding the interaction through Kinect, senior participants had more difficulty interacting with this device, because it demanded a higher body performance. To use this device, it is necessary to move the whole body. The hands are the main parts as they interact with the user interface. It was necessary for the user to stand in front of the device; sometimes it was necessary for the user to move around the device so that it could capture his/her movements. It has to be considered that movement coordination was not optimal during the session, even vision and objective perception led the user to achieve a low performance. Kinect was the second device placed in user acceptance. It is advised to take into account the physical conditions and deterioration of motor abilities, vision and hearing of senior users for the development of new video games with this type of interactive technology.

Concerning interaction through Wii, this device produced low levels of performance, that is, interaction was not favorable. The use of this device was complicated for seniors because it was difficult to hold with one hand, aiming and pressing the fire/shooting button. This is the reason why better coordination was required also having a pulse control while holding the arm up. Moreover, the need to keep the button pressed to make a movement was observed; therefore, elderly users must hold their arm at a certain height, press the button and make a move, all at the same time. Some of the participants had the impulse to hold the control with both hands. Also, this device obtained a low performance level in effective throws. Wii was the control device with less effective throws and most missed throws. It is necessary to analyze interaction mechanisms with the interface because they are too rigid; the user needs more room to maneuver.

Regarding interaction through the Touchscreen, the device did not present considerable difficulty to execute the game. In contrast to other devices, it did not require body movement coordination or pulse control, only visual and touch. However, game interaction required the use of only one finger to touch the screen and, this implies, keeping the arm raised for moments. This is an important observation because using a touchscreen makes it is easy to locate it at the user's sight level, which in turn favors and makes easier for the participant to have a complete panorama and sight of the game, and thus make an effective throw/shot. Moreover, through touchscreen devices, games can be adapted to play musical instruments, skill games, pairs, checkers, domino, among others; for instance, Microsoft Surface has been used to help in the treatment of children with cerebral palsy, as the Surface device uses an interactive table which is programmed with personalized games 
[20]. This device does not require a high level of body movement, though it can demand a higher mental agility.

Finally, regarding the interaction with Xbox control, the Xbox device obtained the highest score. Xbox demanded body (arms, hands, fingers), visual and mental coordination. While playing, the user must visualize the buttons in the control and the game on the screen and, then, calculate the throw/shot. Xbox was the most comfortable device and the one most demanded. The weakest point of Xbox, however, is that it does not require movement from the whole body, even though Xbox offers a device to be used with both hands. The strongest point is its cognitive development: Developing new mental videogames which help in multitasking functions, such as Neuro Racer [6], could be an interesting topic.

\section{Discussion}

The original research question of the research reported in this paper was What is the perception of Usability of seniors using different types of control devices in video games? As in the study reported in [8] where seniors mentioned that "It is very easy to learn to play the game," "I enjoy the challenge, I lose the feeling of self-consciousness during the process of recalling," results indicate that seniors participating in the present study (Study 1) using Kinect expressed comments such as “... Well, it wasn't too difficult for me, it was easy..." and also "... $\mathrm{Oh}$, it was too short, but productive because we captured the dragon quickly..." indicating Learnability, and expressed satisfaction indicators such as “... I feel motivated, this is something I didn't know and that I could do without any problem...," “... I am excited because I did in less than 2 minutes; it took us seconds to catch the dragon...," “... I was learning, I was even laughing, I never thought I would be playing...," “... When you are having a great time, time flies always so quickly..."

Concerning the results of Efficiency and Errors as usability characteristics, it was concluded that Xbox was the device with which the seniors achieved more effectiveness and the least number of missed throws. Xbox looks like the favorite device to interact with, while Kinect and the Touchscreen showed a similar level of missed throws and the least effective device was Wii.

Regarding effective throws, Kinect is the second device with which participants achieved the most effective throws and Touchscreen was the device with which senior users had higher difficulty to achieve effective throws. As of Effective and Missed Throws, the Xbox averaged the highest effective throws score and Wii the highest missed throws score.
With regard to control time, Xbox offered participants the opportunity to take more time on calculating movements and take control over the game, so that the participant invested part of that time on how his/her movement would be and applying it. As Xbox allowed more time so that a participant could decide the moment to make a play (throw), this indicates that time invested allows a higher probability to hit and obtain an effective throw. Thus, with this device participants achieved a better performance.

Therefore, it is assumed that control time has a direct influence on the throw, that is, a longer control time means more effective throws. Kinect and Wii have similarities when compared; both of them did not require long control time, which is why it is inferred that there are higher probabilities for missed throws. However, it is important to emphasize that the Wii and Kinect controls had more missed throws and spent more control time. In contrast, the Touchscreen registered less time for the control time with less effective throws.

Thus, indicators of ease of use (learnability) and satisfaction with the Kinect device have been identified (Study 1). Concerning Efficiency and Errors, the Xbox device showed less missing throws and more effective throws.

Although this is a pilot study and the results are preliminary, it intends to explore and address a significant gap in terms of research on the usability perception of different videogame devices for senior users. It would be important to add a follow-up group of participants to verify the memorability characteristic, which can be obtained by considering the time required to develop a task previously experimented with [8].

It is important to replicate this study, though an experimental research design that provides greater control and isolates the effect of various foreign variables, thus reducing alternative explanations, should be considered. A research design with both a waiting list control group and an experimental group (with two or more conditions) can be ideal for monitoring studies of this nature. It is also recommended that these characteristic studies be performed with a greater number of participants to verify usability indicators with other technology.

\section{Conclusions}

The video game control must facilitate and allow an adequate time for the senior user to make decisions to achieve the objectives in the game.

In this sense, it must be noted that Xbox did not demand too much body coordination, apart from eye and finger coordination. This device was easy to use as the user had it at sight level and felt confident because it responded quite 
well (control). Also, the device's stick and buttons were the easiest to control, because they were very accessible.

Regarding the Touchscreen, it was easy to aim; however, the throw/shot was not easy to control because this device was too sensitive to the movement of the hand. This type of device has a disadvantage because the arm has to be raised and it causes fatigue to the user.

Regarding Kinect, the game required greater body coordination. Also, Kinect causes fatigue as the game demanded the user to be on his/her feet and keep his/her arms above the head.

Regarding Wii, it was difficult for senior users to coordinate their fingers to the movement of their hands, as they needed to use one hand and, at the same time, press a button, and this device requires a higher level of coordination. It was complicated for seniors to hold and aim with this type of device.

Given the aforementioned, it is important to ensure that the senior user is willing to use his/her time and energy to play videogames; further studies are suggested to understand the motivation and needs of seniors to play. Using more creative and user-centered methodologies could offer insight into what senior users needs and want, and how they are willing to use new technology.

Open Access This article is distributed under the terms of the Creative Commons Attribution 4.0 International License (http://crea tivecommons.org/licenses/by/4.0/), which permits unrestricted use, distribution, and reproduction in any medium, provided you give appropriate credit to the original author(s) and the source, provide a link to the Creative Commons license, and indicate if changes were made.

\section{References}

1. Goldstein, J., Cajko, K., Oosterbroek, M., Michielsen, M., van Houten, O., Salverda, F.: Video games and the elderly. Soc. Behav. Personal. 25(4), 345-352 (1997)

2. Wollersheim, D., Merkes, M., Shields, N., Liamputtong, P., Wallis, L., Reynolds, F., Koh, L.: Physical and psychosocial effects of Wii video game use among older women. Int. J. Emerg. Technol. Soc. 8(22), 85-98 (2010)

3. Hwang, M., Hong, J., Hao, Y., Jong, J.: Eldeŕs usability, dependability, and flow experiences on embodied interactive video games. Educ. Gerontol. 37, 715-731 (2011)

4. Rute-Pérez, S., Santiago-Ramajo, S., Hurtado, M., RodríguezFórtiz, M., Caracuel, A.: Challenges in software applications for the cognitive evaluation and stimulation of the elderly. J. NeuroEng. Rehabil. 11(1), 1-10 (2014). doi:10.1186/1743-0003-1188
5. Miesenberger, K., Klaus, J., Zagler, W., Karshmer, A. (eds.): Computers helping people with special needs. Lecture Notes in Computer Science, vol. 6180. Springer, Switzerland (2010)

6. Coyne, K., Nielsen, J.: Beyond Alt Text: Making The Web Easy to Use for Users with Disabilities. Nielson Norman Group, Fremont (2001)

7. Fisk, A., Rogers, W., Chamess, N., Czaja, S., Sharti, J.: Designing For Older Adults: Principles and Creative Human Factors A Roaches. CRC, Boca Raton (2004)

8. Rubin, J., Chisnell, D.: Handbook of Usability Testing: How to Plan, Design, and Conduct Effective Tests, 2nd edn. Wiley, New York (2008)

9. Hwang, M., Hong, J., Hao, Y., Jong, J.: Elders' usability, dependability, and flow experiences on embodied interactive video games. Educ. Gerontol. 37(8), 715-731 (2011)

10. Theng, Y.-L., Teo, P., Truc, P.: Investigating sociability and affective responses of elderly users through digitally-mediated exercises: a case of the Nintendo Wii. In: Forbrig, P., Paternó, F., Mark Pejtersen, A. (eds.) Human-Computer Interaction, vol 332. IFIP Advances in Information and Communication Technology, pp. 152-162. Springer, Berlin (2010). doi:10.1007/978-3-64215231-3_16

11. Rubin, J.: Handbook of Usability Testing: How to Plan, Design, and Conduct Effective Tests. Wiley, New York (1994)

12. Schweiger, I., Czabke, A., Lueth, T.: An elderly-oriented platform to simplify the use of physical activity controlled game consoles. In: Wichert, R., Eberhardt, B. (eds.) Ambient Assisted Living, pp. 91-100. Springer, Heidelberg (2011). doi:10.1007/ 978-3-642-18167-2_7

13. Rice, M., Carmichael, A.: Effective requirements gathering for older adults. SIGACCESS Access. Comput. 88, 15-18 (2007). doi: $10.1145 / 1278234.1278237$

14. Lankoski, P., Heliö, S., Nummela, J., Lahti, J., Mäyrä, F., Ermi, L.: A case study in pervasive game design: the songs of north. Paper presented at the proceedings of the 3 rd Nordic conference on Human-computer interaction, Tampere (2004)

15. Blythe, M., Wright, P., Bowers, J., Boucher, A., Jarvis, N., Reynolds, P., Gaver, B.: Age and experience: ludic engagement in a residential care setting. Paper presented at the proceedings. DIS'08, (2010)

16. Barrington, L., Lyons, MJ., Diegmann, D., Abe, S.: Ambient display using musical effects. Paper presented at the proceedings of the 11th international conference on Intelligent user interfaces, Sydney, (2006)

17. Kerlinger, F., Lee, H.: Foundations of Behavioral Research. Cengage Learning, Orlando (1999)

18. ControlFreakStudio. HappySky. https://www.facebook.com/ media/set/?set=a.373332556112564.1073741829.133072416805 247 (2011)

19. Corbin, J., Strauss, A.: Basics of Qualitative Research: Techniques and Procedures for Developing Grounded Theory. SAGE Inc, Thousand Oaks (2008)

20. Ostrovsky G (2010) Touchscreen gaming for cerebral palsy rehabilitation. http://www.medgadget.com/2010/11/touchscreen_ gaming_for_cerebral_palsy_rehab.html 